\title{
Aparato experimental para o ensino de tópicos da eletrostática: o eletroscópio com transistor de efeito de campo ${ }^{+*}$
}

\author{
Thiago Alves de Sá Muniz Sampaio ${ }^{1}$ \\ Eriverton da Silva Rodrigues ${ }^{2}$ \\ Cícero Jailton de Morais Souza ${ }^{3}$ \\ Instituto Federal de Educação, Ciência e Tecnologia do Sertão Pernambucano \\ Campus Salgueiro \\ Salgueiro - PE
}

\section{Resumo}

Os laboratórios escolares tradicionais carecem de experimentos que possam identificar claramente muitos dos fenômenos presentes no estudo da eletrostática. Este trabalho tem como proposta a inserção de um novo tipo de aparato experimental simples para o ensino de tópicos desta área da física, consistindo em uma versão de eletroscópio que utiliza o transistor de efeito de campo para a detecção de cargas elétricas provenientes de corpos eletrizados. É feita uma explanação sobre os princípios que tornam este tipo de transistor um dispositivo eficaz devido à sua alta sensibilidade aos campos eletrostáticos, além de uma análise sobre a utilidade deste projeto para a visualização de muitos fenômenos peculiares, como a polarização e a indução. Com base nisso, propomos algumas atividades simples que podem ser realizadas em sala de aula a fim de envolver os alunos nos conteúdos iniciais da eletrostática. Temos a perspectiva de que essa forma de ensino associada à experimentação e demonstração dos fenômenos em sala de aula possa promover uma melhor aprendizagem destes conceitos por parte dos alunos, demonstrando a utilidade da experimentação para o ensino de eletrostática.

\footnotetext{
${ }^{+}$Experimental apparatus for teaching electrostatic topics: the electroscope with field-effect transistor

* Recebido: maio de 2016.

Aceito: novembro de 2016.

1 E-mail: alveshere@ hotmail.com

2 E-mail: erivertonr@hotmail.com

3 E-mail: cicero.jailton@hotmail.com
} 
Palavras-chave: Ensino de eletrostática; Corpos eletrizados; Física experimental.

\begin{abstract}
Regular school labs lack experiments that can properly identify many of the phenomena present in the electrostatic study. This paper proposes the implementation of a new kind of simple experimental apparatus for teaching topics in this area of Physics, consisting in a kind of electroscope that uses the field-effect transistor for detecting electric charges coming from electrified bodies. An explanation is given on the principles that make this type of transistor an effective device due to its high sensitivity to electrostatic fields, as well as an analysis of the usefulness of this project for viewing many peculiar phenomena, such as polarization and induction. Based on this, we propose some simple activities that can be done in the classroom to involve students in the initial subject of electrostatics. We expect that this form of teaching along with experimental and explanatory approach of the phenomena in the classroom can bring to students a better learning of these concepts, demonstrating the utility of experimentation on teaching electrostatics.
\end{abstract}

Keywords: Electrostatic teaching; Electrified bodies; Experimental Physics.

\title{
I. Introdução
}

Desde as primeiras observações e estudos sistemáticos realizados por diversos cientistas nos séculos XVII e XVIII, pode ser verificado que a compreensão dos fenômenos eletrostáticos sempre esteve intimamente ligada com a experimentação. Praticamente todo o desenvolvimento teórico obtido por esta ciência foi construído à partir desse prisma. O caráter instigante que a eletricidade possui está intrinsecamente relacionado com o seu caráter experimental, isto é, com a observação direta e com a dedução das aparentes causas e consequências de fenômenos, que vão desde a força de atração que um corpo carregado por atrito promove em objetos leves até a ocorrência de raios em uma tempestade. Isso ressalta a importância de estabelecer um tipo de abordagem voltada ao uso de laboratórios didáticos ao ensinar esses conteúdos na escola ou na universidade, como é reforçado por Alves (2000).

Quanto ao ensino desses conteúdos, é notório que os fenômenos eletrostáticos constituem a base para a compreensão do eletromagnetismo como um todo, visto que é nessa fase que os conceitos de carga e campo elétrico são abordados pela primeira vez. Para a explicação 
de tais conceitos em sala de aula, torna-se indispensável o uso da experimentação concomitantemente à aulas expositivas. Muitos autores ressaltam a importância de se realizar atividades experimentais ao se ensinar física ou ciências em geral. Podemos destacar Séré et al. (2004), Araujo e Abib (2003), Giordan (1999), Arruda e Laburú (1998). Além disso, atividades experimentais de eletrostática podem promover uma interrelação com todo o desenvolvimento histórico desta ciência, como é abordado por Silva (2011).

No que diz respeito à visualização prática destes fenômenos, muitos laboratórios escolares carecem de experimentos que possam demonstrar de maneira efetiva alguns fenômenos que ocorrem com a eletrização de corpos. $\mathrm{O}$ experimento mais comumente encontrado nesses laboratórios é o eletroscópio de folhas metálicas, representado na Fig. 1.

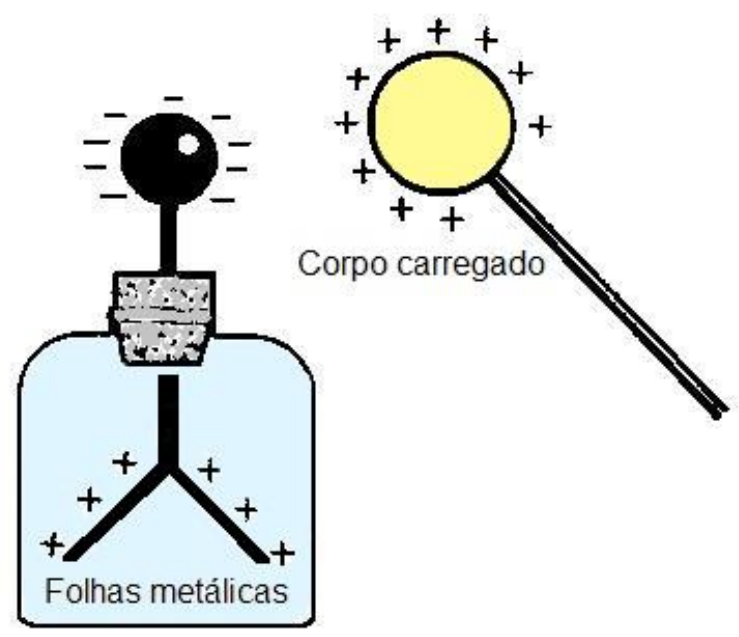

Fig. 1 - Representação de um eletroscópio de folhas metálicas. Um corpo carregado é aproximado e induz cargas de mesmo sinal nas folhas, fazendo elas se repelirem.

Ao aproximarmos um objeto carregado deste eletroscópio, nota-se o efeito de repulsão entre as folhas metálicas, que por conta da indução eletrostática ganham cargas de mesmo sinal, sendo este sinal igual ao da carga do objeto. Porém, com quantidades baixas de carga a repulsão entre as folhas não é muito nítida, o que dificulta a visualização de certos fenômenos. Outro ponto importante é que com este eletroscópio não conseguimos saber a polaridade da carga que o objeto apresenta (a menos que o eletroscópio já se encontre eletrizado com uma carga de sinal conhecido) (MÁXIMO; ALVARENGA, 2011, p. 24).

Uma versão eletrônica de eletroscópio pode ser feita com o uso de um transistor de efeito de campo (FET - field-effect transistor). Este tipo de eletroscópio apresenta uma sensibilidade muito maior do que o eletroscópio tradicional de folhas na detecção de campos eletrostáticos, facilitando bastante a visualização de certos fenômenos peculiares da eletrostática. Neste trabalho, explicamos os princípios físicos que permitem o FET ser utilizado com este propósito e mostramos como alguns desses fenômenos podem ser abordados em sala de aula utilizando um projeto de eletroscópio simples à base de FET. 


\section{O transistor de efeito de campo}

\section{II.1 Princípio de mecanismo do FET}

O FET se caracteriza basicamente como um dispositivo amplificador, e por isso é largamente utilizado em projetos para amplificação de sinal em geral. Ele apresenta três terminais, denominados Porta, Dreno e Fonte, que são normalmente designados pelas letras G, D e S, respectivamente (do inglês, gate, drain e source). Esse transistor apresenta uma importante diferença em relação ao tradicional transistor bipolar de junção (TBJ). Enquanto o TBJ é um dispositivo controlado por corrente, o FET é um dispositivo controlado por tensão (diferença de potencial) (BOYLESTAD; NASHELSKY, 2004, p.174). A corrente que passa entre o emissor e o coletor do TBJ é controlada pela corrente de entrada no terminal da base, já para o FET a diferença de potencial entre os terminais da Porta e Fonte controla a corrente que passa entre a Fonte e o Dreno (MALVINO, 1997, p. 552).

Esses transistores são constituídos de junções p-n. Essas junções são feitas através de dopagem de um material do tipo $\mathrm{p}$ (material com escassez de elétrons livres) sobre a superfície polida de um semicondutor do tipo n (material com excesso de elétrons livres), ou vice-versa. O FET de canal n (que é o mais facilmente encontrado) é constituído de um material semicondutor ao qual são ligados os terminais S e D. O terminal G é ligado internamente em um material do tipo n. As junções p-n do material semicondutor que compõem o canal são ligados externamente ao terminal $\mathrm{G}$.

Existem basicamente dois tipos de FET: o FET de junção (ou JFET) e o FET de Porta isolada (ou IGFET), às vezes denominado de MOSFET (sigla de metal-oxid-semiconductor field-effect transistor - 'transistor de efeito de campo metal-óxido-semicondutor') (YOUNG e FREEDMAN, 2009b, p. 318). A diferença básica entre esses dois tipos é o modo como as regiões n ou p são conectadas no interior do dispositivo.

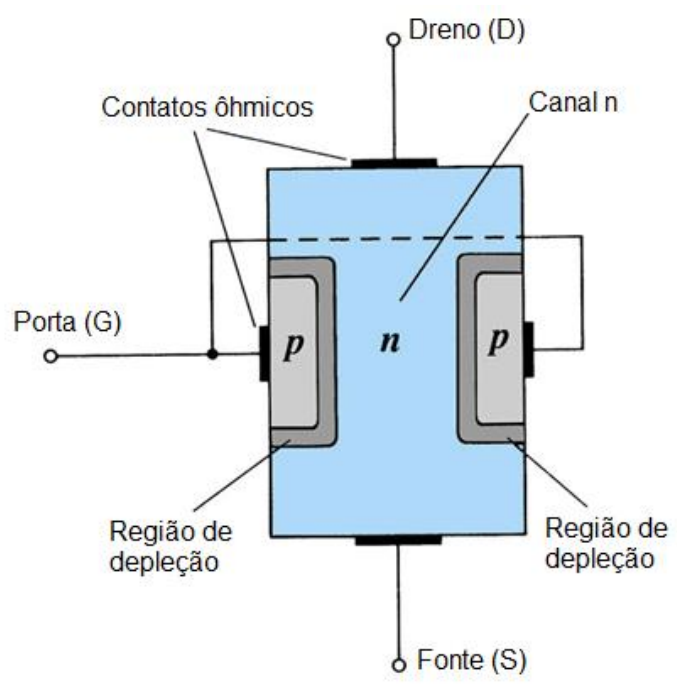

Fig. 2 - Diagrama de um JFET de canal n. 
Na Fig. 2 é mostrado um diagrama básico de um JFET semelhante ao usado no projeto do eletroscópio proposto neste artigo. A região central deste transistor é composta essencialmente de material do tipo n. Nesta região são conectados através de contatos ôhmicos os terminais do Dreno (D) e da Fonte (S). O terminal da Porta $(\mathrm{G})$ é conectado externamente às duas regiões do tipo p. Para estabelecer o sentido convencional do fluxo de elétrons entrando pelo terminal da Fonte e saindo pelo Dreno, deve-se ligar o terminal da Fonte no terminal negativo da fonte de alimentação, e o Dreno, por sua vez, no terminal positivo (a inversão desta ligação implicará apenas na mudança do sinal da corrente).

Ao se estabelecer uma diferença de potencial $V_{D S}$ entre o Dreno e a Fonte, deverá aparecer uma corrente elétrica IDS atravessando o canal $n$. A largura efetiva do canal $n$ por onde passam os elétrons é diretamente controlada pelas regiões de depleção presentes nas junções pn (REZENDE, 2015, p. 204).

\section{II.2 Corpos eletrizados nas proximidades}

Quando a diferença de potencial $V_{G S}$ entre a Porta e a Fonte for igual a zero e quando a tensão $\mathrm{V}_{\mathrm{DS}}$ entre a Fonte e o Dreno for maior que zero, a corrente $\mathrm{I}_{\mathrm{DS}}$ manterá um valor estável (denominado corrente de saturação). Porém, ao estabelecer valores negativos para $\mathrm{V}_{\mathrm{GS}}$ (o que equivale a um corpo com carga negativa próximo ao terminal da porta), o valor de IDs irá diminuir. Isto ocorre porque, ao colocar a Porta em um potencial negativo em relação ao potencial da Fonte, haverá uma polarização reversa nas junções p-n. Tudo acontece da seguinte maneira: quando há carga negativa no terminal da Porta, esta repele a carga negativa presente na região p. Há poucos elétrons nessas regiões, mas mesmo assim são suficientes para aumentar as regiões de depleção em torno do canal n (Fig. 3). Dito de outro modo, o campo elétrico resultante desta polarização irá dificultar a passagem dos elétrons no canal, resultando em uma maior resistência e, portanto, uma menor corrente IDs.

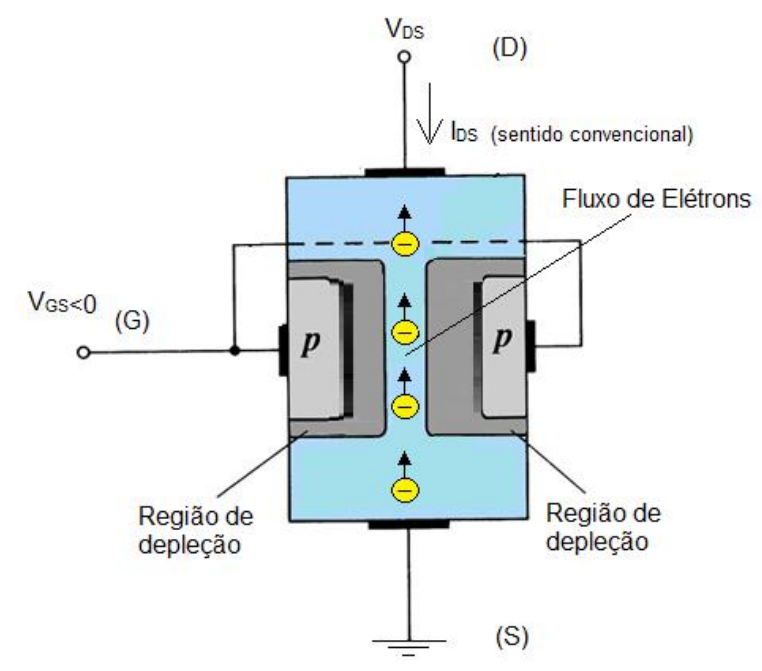

Fig. 3 - JFET de canal $n$ reversamente polarizado: corpo com cargas negativas nas proximidades da porta. Aumento da região de depleção e diminuição da corrente $I_{D S}$. 
Ao se estabelecer valores positivos para $\mathrm{V}_{\mathrm{GS}}$ (corpo carregado positivamente próximo à Porta), a Porta estará submetida a um potencial positivo em relação à Fonte. Na prática, isto irá polarizar diretamente as junções p-n do dispositivo, fazendo diminuir então a região de depleção no canal (Fig. 4). Isso irá corresponder a uma diminuição da resistência e um aumento do valor de $\mathrm{I}_{\mathrm{DS}}$.

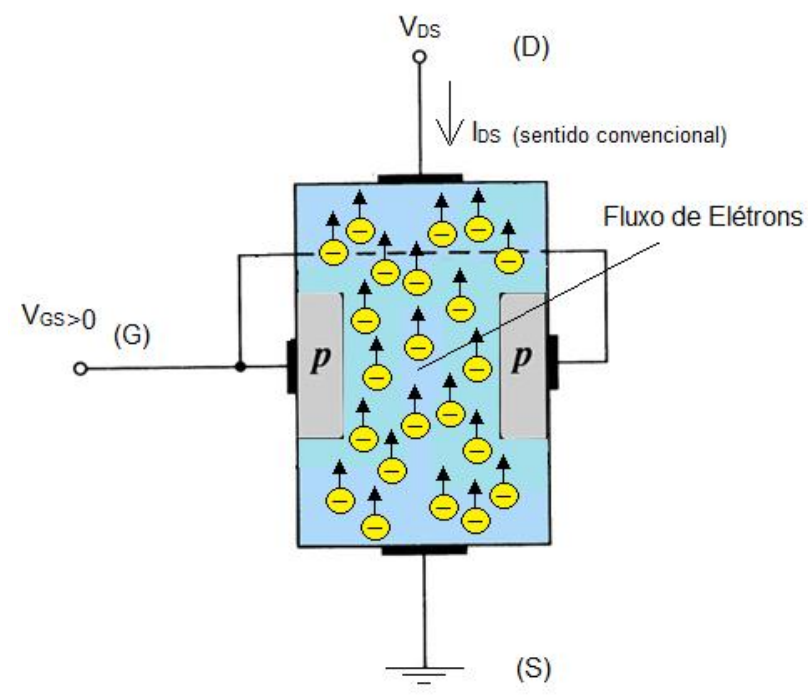

Fig. 4 - JFET de canal $n$ diretamente polarizado: corpo com cargas positivas nas proximidades da porta. Diminuição da região de depleção e aumento da corrente IDS.

Portanto, para ambos os casos, o valor de IDS apresenta variações bastante evidentes mediante às pequenas variações de potencial na porta, o que torna este dispositivo extremamente eficiente para a deteç̧ão de corpos carregados nas proximidades.

\section{O eletroscópio}

O projeto de eletroscópio proposto preza pela facilidade na montagem e pela fácil aquisição dos componentes, que podem ser encontrados em lojas de eletrônica básicas. Foram testados diversos protótipos para o projeto, de modo a chegarmos em uma versão final que satisfizesse as propostas didáticas na visualização dos fenômenos eletrostáticos.

\section{III.1 Montagem e materiais}

Para a montagem do projeto foram usados um transistor de efeito de campo MPF102 (ou BF245); dois resistores (1M $\Omega$ e $1 \mathrm{~K} \Omega$ ); um LED; um multímetro; fio de cobre rígido; placa para protótipos de circuitos e bateria de $9 \mathrm{~V}$. A conexão dos componentes se deu de acordo com o diagrama da Fig. 5. 


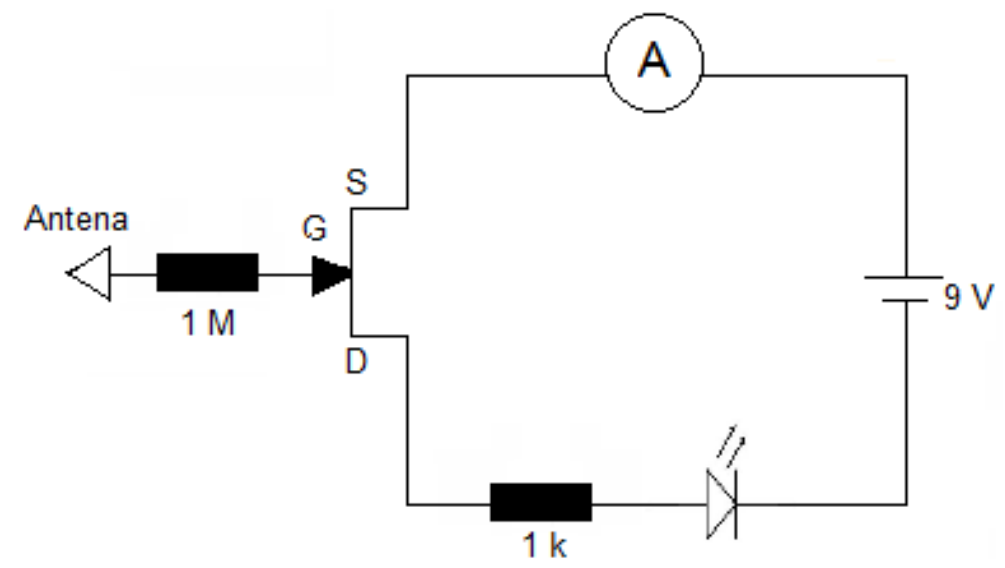

Fig. 5 - Diagrama do circuito do eletroscópio.

É importante ressaltar que o eletroscópio não funciona de maneira adequada com a utilização de fontes de alimentação ligadas à rede doméstica, pois os componentes das fontes retificadoras modificam a forma como a corrente varia no circuito.

\section{III.2 Funcionamento}

O fio de cobre atuará como uma antena, aumentando a sensibilidade na detecção de campos eletrostáticos. É preferível que o fio usado seja esmaltado, para evitar toques acidentais no cobre, visto que uma pessoa pode facilmente acumular cargas estáticas elevadas que poderiam danificar o transistor caso houvesse contato direto com a Porta. Quanto ao comprimento do fio, em geral, $15 \mathrm{~cm}$ já é o suficiente para detectar cargas de objetos eletrizados a uma distância de 1 a 2 metros, porém isto irá depender de alguns fatores como, por exemplo, a umidade do ar. Em dias chuvosos onde a umidade do ar está relativamente alta, a troca de cargas entre corpos atritados se torna mais difícil, e um corpo carregado se descarrega rapidamente devido a uma fina camada de água que o envolve, tornando-o condutor (NUSSENZVEIG, 1997, p. 4). Isto pode dificultar até certo ponto a visualização de alguns fenômenos.

O resistor de $1 \mathrm{M} \Omega$ serve apenas para proteger o transistor no caso de haver potenciais relativamente altos na Porta (o que pode ocorrer se for utilizado um gerador de Van De Graff, por exemplo). Nestes casos é recomendável a utilização deste resistor. O multímetro deverá ser ligado na função de amperímetro, visto que irá detectar as variações de carga através do valor da corrente indicada (que neste caso pode ir de 0 até $4.5 \mathrm{~mA}$ ). O LED servirá para facilitar a visualização destas variações. Neste caso a única função do resistor de $1 \mathrm{~K} \Omega$ é proteger o LED da tensão de $9 \mathrm{~V}$. 


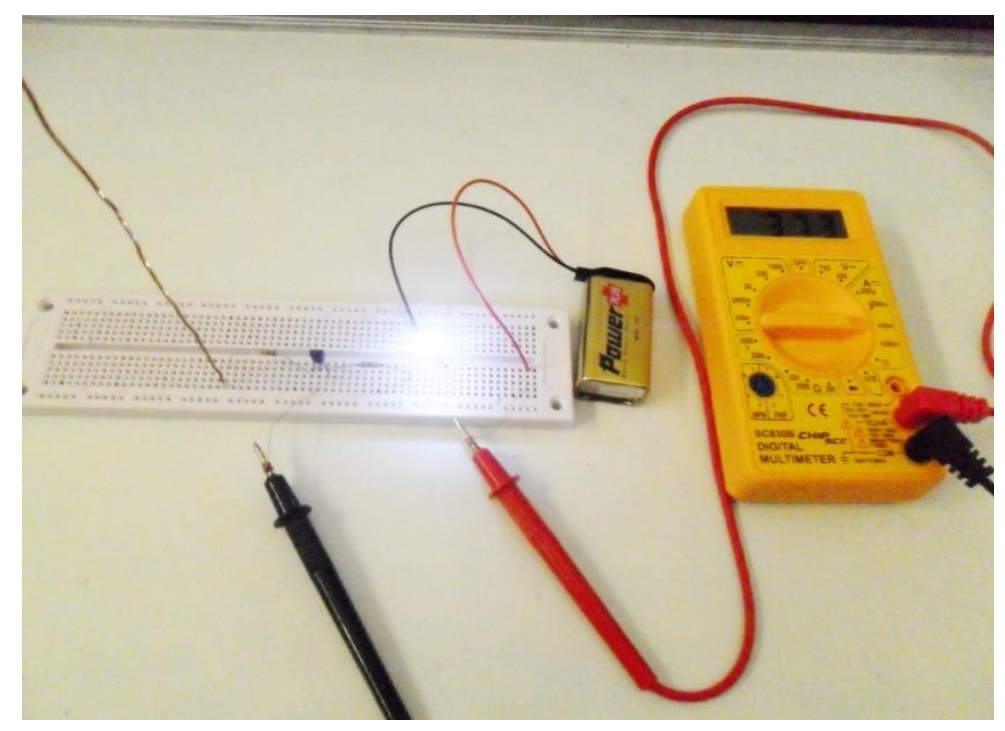

Fig. 6-O eletroscópio em funcionamento.

Inicialmente, quando não há nenhuma carga nas proximidades, a corrente no circuito irá manter um valor estável de equilíbrio. Uma opção para manter o valor da corrente estável na ausência de cargas é conectar algum tipo de aterramento diretamente no terminal da Fonte, isto colocará a Fonte em um potencial zero, de modo que o valor de $\mathrm{V}_{\mathrm{GS}}$ será somente o potencial na Porta. Este aterramento, porém, é opcional, servindo apenas para estabilizar o nível da corrente de equilíbrio, que, para $\mathrm{V}_{\mathrm{GS}}=0$, assumirá o valor da corrente de saturação do FET.

\section{Sugestões para abordagens demonstrativas}

Alguns fenômenos da eletrostática podem ser visualizados através de demonstrações simples utilizando o eletroscópio com FET. As atividades propostas foram verificadas experimentalmente pelos autores. A aplicação dessas atividades em sala de aula pode ser válida tanto no ensino médio como no superior, a depender do grau de formalismo adotado.

\section{IV.1 Polaridade das cargas}

Quando aproximamos um corpo carregado da antena, este irá provocar efeitos diferentes de acordo com a polaridade da carga. Quando a carga do corpo for negativa, a Porta estará submetida a um potencial mais baixo em relação ao da Fonte $\left(\mathrm{V}_{\mathrm{GS}}<0\right)$, o FET estará reversamente polarizado e as regiões de depleção "estreitam" o canal n por onde passam os elétrons. Isto implicará na redução do valor da corrente I IS detectada pelo multímetro, bem como na redução da intensidade do brilho do LED. Quanto menor for a distância do corpo à antena menor será a corrente, de modo que, para um certo potencial, a corrente chegará praticamente a zero e o LED se apagará. À medida que afasta-se o corpo, a corrente voltará ao seu estado de equilíbrio. 
Para cargas positivas, no entanto, o eletroscópio se comporta de maneira completamente diferente. Ao aproximar da antena um corpo carregado positivamente $\left(\mathrm{V}_{\mathrm{GS}}>0\right)$, ocorrerá apenas um pequeno aumento no valor de $\mathrm{I}_{\mathrm{DS}}$ (o brilho do LED pouco varia neste caso), visto que na situação de equilíbrio as regiões de depleção já são pequenas. Para este caso então, o FET se encontra diretamente polarizado (não há regiões de depleção), ou seja, os elétrons (presentes em grande quantidade na região $n$ ) ganham energia suficiente para romper a barreira de potencial nas junções p-n, o que explica a diminuição da resistência interna do dispositivo e o aumento da corrente.

Uma situação peculiar ocorre quando o FET se encontra diretamente polarizado e afastamos o corpo positivo das proximidades da antena. $\mathrm{O}$ valor de IDS irá diminuir bruscamente à medida que afastamos o corpo, podendo este valor chegar próximo a zero dependendo da quantidade de carga do corpo (o LED se apagará). Essa situação pode se manter durante alguns segundos, mesmo sem nenhuma carga nas proximidades. Neste caso, o afastamento do corpo positivo promove uma polarização reversa no FET de canal n. O que ocorre neste caso é que alguns elétrons que percorriam livremente as junções p-n (no caso da polarização direta) agora encontram-se confinados nas regiões p, pois não possuem energia suficiente para retornar à região n. Esses elétrons criam grandes regiões de depleção em torno das junções, promovendo um grande aumento da resistência interna do FET. Depois de alguns segundos, os elétrons vão aos poucos retornando à região $n$ devido às correntes de recombinação geradas através da agitação térmica no interior do dispositivo (EISBERG; RESNICK, 1979, p. 594) fazendo com que acorrente IDS retorne ao seu valor estável.

\section{IV.2 Condutividade}

Devido à alta sensibilidade do instrumento, este pode ser utilizado para evidenciar a condutividade do corpo humano. Se uma pessoa, estando com calçados abertos, friccionar bruscamente um dos seus pés no chão enquanto estiver com as mãos próximas da antena, o atrito do calçado com o chão irá induzir cargas no pé, que são redistribuídas em um fluxo rápido para todo o corpo. Essas cargas tentam escapar pelos braços e mãos, que se encontram esticados (isto evidencia o poder das pontas). A antena então detecta essa mudança de potencial, fazendo a corrente no circuito variar bruscamente (o LED irá piscar). Ao friccionar de novo o calçado, porém desta vez sem as mãos próximas à antena, a corrente no eletroscópio pouco irá variar. Interessantemente, quando o indivíduo que fricciona no chão um dos pés (estando este calçado), enquanto o outro pé permanece no chão descalço, verifica-se que, mesmo com as mãos muito próximas da antena, nada irá ocorrer com o eletroscópio. Isto evidencia o equilíbrio eletrostático proveniente do contato com a Terra, que funciona como uma fonte (ou sumidouro) praticamente inesgotável de elétrons. 


\section{IV.3 Polarização em dielétricos}

Quando aproximamos um objeto carregado de um dielétrico neutro, este induzirá uma distribuição não uniforme das cargas no interior do dielétrico. Os elétrons presentes em um dielétrico se encontram essencialmente ligados aos seus respectivos átomos, no entanto, a presença de um campo eletrostático promove uma redistribuição na simetria de cargas das moléculas do dielétrico, resultando assim em um arranjo de dipolos elétricos induzidos no material. Tal efeito denomina-se polarização (YOUNG; FREEDMAN, 2009a, p. 121; GRIFFITHS, 2011, p. 117).

O eletroscópio permite a visualização da polarização de um dielétrico. Como atividade de demonstração, pode-se colocar um dielétrico (o dielétrico pode ser, por exemplo, uma folha de papel) entre a antena e um objeto carregado (que pode ser um pente de plástico atritado aos cabelos de uma pessoa ou um canudo plástico atritado com papel toalha). A presença da folha de papel entre a antena e o corpo carregado promove uma redução na taxa de variação da corrente, ou seja, a luminosidade do LED varia menos com a presença do corpo carregado, visto que a polarização do dielétrico reduz o campo detectado pela antena. Isto se dá pelo fato de que na superfície da folha de papel voltada para o corpo carregado cria-se um excesso de cargas polarizadas de sinais contrários ao do corpo.

\section{IV.4 Blindagem e gaiola de Faraday}

Em um condutor existe uma quantidade extremamente grande de elétrons livres. Quando um corpo carregado aproxima-se de um condutor ideal, este induzirá uma distribuição de carga na superfície do condutor, sendo que essa carga induzida terá praticamente o mesmo módulo da carga presente no corpo carregado, porém de sinal contrário (GRIFFITHS, 2011, p. 68).

Substituindo-se a folha de papel do exemplo descrito na seção 4.3 por papel alumínio (ou qualquer outro material condutor), praticamente não haverá variação na luminosidade do LED nem variação da corrente, visto que a indução que um corpo carregado promove em um condutor praticamente anula o campo detectado pela antena. Devido à essa blindagem eletrostática, na região entre o papel alumínio e a antena praticamente não existe nenhum campo eletrostático.

Uma atividade interessante pode ser realizada forrando-se as paredes de uma caixa vazia com papel alumínio. O eletroscópio ficará dentro da caixa, deixando-se o multímetro fora dela conectado por meio de uma abertura. Qualquer carga que esteja fora da caixa não será detectada pelo eletroscópio, o dispositivo estará isolado do ambiente externo por uma gaiola de Faraday. Podem-se fazer comparações com o eletroscópio dentro de uma caixa comum, a fim de evidenciar as diferenças entre os fenômenos de polarização nos dielétricos e a blindagem em condutores.

Eletrizando-se a superfície externa da caixa metálica com um gerador eletrostático, podemos verificar que nenhuma carga será detectada pelo eletroscópio no interior da caixa. Isso 
confirma o princípio que diz que qualquer excesso de carga em um condutor fechado em equilíbrio eletrostático irá se concentrar apenas na sua superfície externa.

\section{Perspectivas para o ensino}

Temos a perspectiva de que com este trabalho possa-se estabelecer um melhor aproveitamento no ensino dos conteúdos de eletrostática, partindo do pressuposto que a aprendizagem de tais conteúdos está intimamente ligada com a experimentação em sala de aula. As atividades propostas promovem a visualização de fenômenos que, a princípio, não encontram uma demonstração efetiva em experimentos comuns de laboratórios escolares.

Além disso, as atividades propostas podem ser aplicadas em sala de aula de diferentes maneiras, de acordo com o critério adotado pelo professor. Pode-se, por exemplo, no início da aula, pedir para que os alunos tirem suas próprias conclusões dos fenômenos mostrados, a fim de que participem de modo mais ativo e que simulem a construção do conhecimento científico, reforçando o caráter experimental das atividades. Por outro lado, se houver pouco tempo, as atividades podem ser realizadas com a finalidade de reforçar o conteúdo já visto em sala de aula.

O uso do transistor de efeito de campo nessa fase da aprendizagem permite uma intersecção de áreas diferentes da física (eletrostática e física do estado sólido). Para um completo entendimento das propriedades do FET, pode ser válida a aplicação deste projeto como parte de conteúdos da física moderna, visto que existem efeitos quânticos envolvidos no processo. Isso pode fazer com que alunos do ensino médio ou superior possam encontrar uma aplicação prática de tais conteúdos, além de fornecer conexões com conteúdos prévios.

\section{Referências Bibliográficas}

ALVES, J. P. Regras da transposição didática aplicadas ao laboratório didático. Caderno Catarinense de Ensino de Física, Florianópolis, v. 17, n. 2, p. 174-188, 2000.

ARAÚJO, M. S. T.; ABIB, M. L. V. F. Atividades experimentais no ensino de Física: diferentes enfoques, diferentes finalidades. Revista Brasileira de Ensino de Fisica, v. 25, n. 2, p 176194, 2003.

ARRUDA, S. M.; LABURÚ, C. E. Considerações sobre a função do experimento no ensino de ciências. In: NARDI, R. (Ed.). Questões atuais no ensino de Ciências. São Paulo: Escrituras, 1998. v. 2. p. 53-60.

BOYLESTAD, R. L.; NASHELSKY, L. Dispositivos Eletrônicos e Teoria de Circuitos. 8. ed. Rio de Janeiro: Prentice-Hall do Brasil, 2004. 675 p. 
EISBERG, R; RESNICK, R. Física Quântica - Átomos, Moléculas, Sólidos, Núcleos e Partículas. 4. ed. São Paulo: Campus, 1979. 928 p.

GIORDAN, M. O papel da experimentação no ensino de ciências. Química nova na escola, v. 10, n. 10, p. 43-49, 1999.

GRIFFITHS, D. J. Eletrodinâmica. 3. ed. São Paulo: Pearson Education, 2011. v. 1.

MALVINO, A. P. Eletrônica. 4. ed. São Paulo: Makron Books, 1997. v. 1.

MÁXIMO, A.; ALVARENGA, B. Física. São Paulo: Scipione, 2011. v. 3.

NUSSENZVEIG, H. M. Curso de Física Básica 3 - Eletromagnetismo. São Paulo: Editora Edgard Blucher LTDA, 1997. v. 3.

REZENDE, S. M. Materiais e Dispositivos Eletrônicos. São Paulo: Editora Livraria da Física, 2015. v. 1.

SÉRÉ, M.; COELHO, S. M.; NUNES, A. D. O papel da experimentação no ensino da física. Caderno Brasileiro de Ensino de Física, v. 20, n. 1, p. 31-43, 2003.

SILVA, J. N. Uma abordagem histórica e experimental da Eletrostática. Estação Científica (UNIFAP), v. 1, n. 1, p. 99-113, 2011.

YOUNG, H. D.; FREEDMAN, R. A. Física III: Eletromagnetismo. 12. ed. São Paulo: Pearson Education, 2009. v. 3.

Física IV: Ótica e Física Moderna. 12. ed. São Paulo: Pearson Education, 2009. v. 4. 